\title{
Handcrafted Microwire Regenerative Peripheral Nerve Interfaces with Wireless Neural Recording and Stimulation Capabilities
}

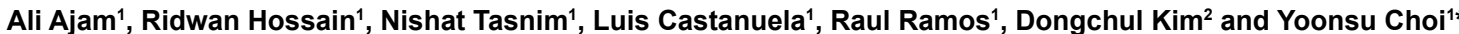

${ }^{1}$ Department of Electrical Engineering, University of Texas Rio Grande Valley, Edinburg, Texas, 78539, USA

${ }^{2}$ Department of Computer Science, University of Texas Rio Grande Valley, Edinburg, Texas, 78539, USA

\begin{abstract}
A scalable microwire peripheral nerve interface was developed, which interacted with regenerated peripheral nerves in microchannel scaffolds. Neural interface technologies are envisioned to facilitate direct connections between the nervous system and external technologies such as limb prosthetics or data acquisition systems for further processing. Presented here is an animal study using a handcrafted microwire regenerative peripheral nerve interface, a novel neural interface device for communicating with peripheral nerves. The neural interface studies using animal models are crucial in the evaluation of efficacy and safety of implantable medical devices before their use in clinical studies. 16-electrode microwire microchannel scaffolds were developed for both peripheral nerve regeneration and peripheral nerve interfacing. The microchannels were used for nerve regeneration pathways as a scaffolding material and the embedded microwires were used as a recording electrode to capture neural signals from the regenerated peripheral nerves. Wireless stimulation and recording capabilities were also incorporated to the developed peripheral nerve interface which gave the freedom of the complex experimental setting of wired data acquisition systems and minimized the potential infection of the animals from the wire connections. A commercially available wireless recording system was efficiently adopted to the peripheral nerve interface. The 32-channel wireless recording system covered 16-electrode microwires in the peripheral nerve interface, two cuff electrodes, and two electromyography electrodes. The 2-channel wireless stimulation system was connected to a cuff electrode on the sciatic nerve branch and was used to make evoked signals which went through the regenerated peripheral nerves and were captured by the wireless recording system at a different location. The successful wireless communication was demonstrated in the result section and the future goals of a wireless neural interface for chronic implants and clinical trials were discussed together.
\end{abstract}

Keywords: Neural interface; Microchannel scaffold; Nerve regeneration; PDMS

\section{Introduction}

Neural interface technologies are envisioned to facilitate direct connections between the nervous system and external technologies such as limb prosthetics or data acquisition systems for further processing. Although cultured in vitro neuronal networks have shown a variety of mechanisms of neuronal functionality, the major role of behavioral control by the nervous system cannot be incorporated with the in vitro neuronal networks system. Neuronal interface signals captured from awake, freely behaving animals are crucial for the next level of clinical applications. In amputees, such technologies would provide direct neural control of prosthetic movements and restore sensory feedback by functionally reconnecting damaged efferent motor and afferent sensory pathways. The peripheral nerve has been one target for bidirectional interfacing, with renewed interest generated by reports that peripheral nerve tissue is viable for interfacing even years after injury or amputation [1-4]. Several designs, such as cuff electrodes, flat interface nerve electrodes (FINE) [5-7], longitudinal intrafascicular electrodes (LIFE) [5,8-10], Utah Slanted Electrode Arrays (USEA) [11-13], and regenerative sieve and microchannel electrodes [14-20] demonstrated selective recording and stimulation. However, the devices have limited electrode sites and recordings can only be obtained from the limited number of nerve fascicles.

A regenerative peripheral nerve interface, developed here, can be utilized to address these goals and is designed to communicate with the brain through the peripheral nervous system. Previously, we developed 4-electrode and 8-electrode microwire regenerative peripheral nerve interfaces ( $\mu \mathrm{PNI}$ ) [21-26]. Here we report an advanced generation of the 16 -electrode $\mu$ PNI and even further advanced the $\mu$ PNI with wireless communication capabilities. The whole implantable microdevice consists of a $\mu \mathrm{PNI}$ for recording placed on the transection site of the sciatic nerve, and three $\mu$ Cuff electrodes, one for stimulation placed on the proximal site of the transection site of the sciatic nerve and the other two for recording placed on the tibial nerve and the common peroneal nerve. Additionally, two electromyography electrode pairs were implanted on the tibialis anterior (TA) and soleus (SOL) muscles on the right hind leg to record the muscle signals during animal's locomotion tests. It gives us the capability of both electrophysiological recording and stimulation to develop a communication pathway from the brain to the endings of peripheral nerves. Peripheral nerve stimulation from one end of the $\mu \mathrm{PNI}$ initiates a neural signal pathway. Animal locomotion on a treadmill was tested in the animal facility at UTRGV and the $\mu$ PNI has enabled us to analyze any sophisticated behavioral patterns.

There are three fundamental neuroscience backgrounds correlated with the $\mu$ PNI (Table 1). Independent microchannel neural interfaces will be creatively achieved by microwires embedded inside the microchannel scaffolds which can be occupied by regenerated nerve

*Corresponding author: Yoonsu Choi, Department of Electrical Engineering University of Texas Rio Grande Valley, Edinburg, Texas, 78539, USA, Tel: 9566657822; E-mail: yoonsu.choi@utrgv.edu

Received December 10, 2015; Accepted January 06, 2016; Published January 13, 2016

Citation: Ajam A, Hossain R, Tasnim N, Castanuela L, Ramos R, et al. (2016) Handcrafted Microwire Regenerative Peripheral Nerve Interfaces with Wireless Neural Recording and Stimulation Capabilities. Sensor Netw Data Commun 5: 133. doi:10.4172/2090-4886.1000133

Copyright: (C) 2016 Ajam A, et al. This is an open-access article distributed unde the terms of the Creative Commons Attribution License, which permits unrestricted use, distribution, and reproduction in any medium, provided the original author and source are credited. 
Citation: Ajam A, Hossain R, Tasnim N, Castanuela L, Ramos R, et al. (2016) Handcrafted Microwire Regenerative Peripheral Nerve Interfaces with Wireless Neural Recording and Stimulation Capabilities. Sensor Netw Data Commun 5: 133. doi:10.4172/2090-4886.1000133

Page 2 of 5

\begin{tabular}{|c|c|}
\hline $\begin{array}{c}\text { Neuroscience } \\
\text { Fundamentals }\end{array}$ & $\boldsymbol{\mu P N I}$ \\
\hline $\begin{array}{c}\text { Peripheral nerves regenerate } \\
\text { (like hair and nails) }\end{array}$ & $\begin{array}{c}\text { Custom designed Microchannel Scaffolds support } \\
\text { nerve regeneration }\end{array}$ \\
\hline $\begin{array}{c}\text { Action potentials are } \\
\text { recordable every } 1 \mathrm{~mm} \text { from } \\
\text { nodes of Ranvier }\end{array}$ & $\begin{array}{c}1.0 \mathrm{~mm} \text { length open microwire Electrodes in } 3 \\
\mathrm{~mm} \text { length microchannel scaffolds }\end{array}$ \\
\hline $\begin{array}{c}\text { CNS-PNS neurons are } \\
\text { connected from the brain to } \\
\text { peripheral nerves }\end{array}$ & $\begin{array}{c}\text { Peripheral Nerve Interface will collect details of } \\
\text { the Brain making the process Noninvasive Brain- } \\
\text { Machine Interfaces }\end{array}$ \\
\hline
\end{tabular}

Table 1: Supports from fundamental neuroscience.

and develop an isolated neural signal communication. Along with peripheral nerve regeneration, the microwires on the nodes of Ranvier can cover and record neural signals selectively from an isolated neural signal source. The microchannel and microwire are long enough to cover and record neural signals from the isolated nerve branch by structural selectivity during nerve regeneration.

\section{Methods}

\section{Fabrication of PDMS scaffolds}

Microfluidic channel scaffolds were developed to direct peripheral nerve growth. 50 wires $(160 \mu \mathrm{m}$ in diameter) were tightly packed into Silastic tubes (OD $1.96 \mathrm{~mm}$, ID $1.47 \mathrm{~mm}$; Cat. No. 508-006, Dow Corning, MI) and then were cast in liquid PDMS (Sylgard ${ }^{\circ}$ 184, Dow Corning, MI) with a 10:1 base to curing agent ratio. They were placed in a vacuum chamber until all air dissipated and then were placed in an oven for 2 hours at $90^{\circ} \mathrm{C}$ to allow the liquid PDMS to solidify. The Silastic tube and Sylgard $184^{\circ}$ are composed of the same PDMS material and became a single structure as the liquid PDMS solidified. The solidified PDMS was soaked in chloroform, causing it to expand. The wires were henceforth, removed leaving behind a long, flexible scaffold with an array of microchannels within it. Chloroform is a highly volatile solvent, leaving no residue when it is evaporated. No special process is required for the fabrication process to clean chloroform. Chloroformswollen PDMS was switched into 70\% ethanol to clean the device while shrinking down PDMS and run sterilization process together. Then PDMS scaffolds were placed in an oven at $100^{\circ} \mathrm{C}$ for 20 minute to make any remaining Chloroform evaporate.

\section{Embedding microwires}

The $75 \mu \mathrm{m}$ diameter microwires (Stablohm 800A, California fine wire, Grover Beach, CA) were inserted in the $160 \mu \mathrm{m}$ diameter microchannels to record the neural signals from the regenerated nerves inside microchannels. No special micromachining equipment was required and commercially available microwires were efficiently used to implement the $\mu$ PNI structures. Once the PDMS scaffolds were fabricated, commercially available microwires $(75 \mu \mathrm{m}$ diameter) were embedded within their microchannels. The scaffold was cut 3 $\mathrm{mm}$ lengthwise and PDMS tubes, used as suture guides, were placed at both proximal and distal ends of the scaffold, hereafter referred to as proximal and distal tubes, respectively. The proximal tube was cut 3.5 $\mathrm{mm}$ long and placed on one end of the scaffold, thereby covering 1.4 $\mathrm{mm}$ of the scaffold. It was secured to the scaffold by placing a drop of liquid PDMS solution where they make contact (on the outer surface of the scaffold) and placing in an oven at $90^{\circ} \mathrm{C}$ for 10 minutes. The distal tube was cut $4 \mathrm{~mm}$ long and placed so that it covered $1.4 \mathrm{~mm}$ of the other end of the scaffold. To assist in the process of embedding microwires in the scaffold, a small circle with a slit leading to it was made in the distal tube. The gap between the tubes was filled with a dental cement and was subjected to ultraviolet (UV) light for 8 seconds to cause it to harden. The distal tube was opened along the slit to facilitate embedding of microwires into the microchannel.

16 microwires were cut into 8 inch segments and $1 \mathrm{~mm}$ of insulation was trimmed off at the tips. They were then folded at $90^{\circ}$ angles, $1 \mathrm{~mm}$ away from the uninsulated parts. The exposed wires were placed oneby-one into the microchannels through the circle made in the proximal tube and glued to the dental cement previously applied using the same technique. Dental cement was then used to seal the circle in the proximal tube, being careful not to allow any dental cement into the regenerating path of axons. All 16 wires were then braided together to make them as compact as possible and were connected subcutaneously to a head stage connector which was attached to the skull. Microwires are beneficial because they are easy to implant, permit smaller wounds, and create minimal obstruction to the regenerative path.

\section{Animal implantation}

Surgical procedures were performed under aseptic conditions at the UTRGV Animal Facility (Figure 1). Prior to implantation, a Lewis rat was placed into an induction chamber and subjected to gas anesthesia (Isoflurane) until unconscious. The surgery locations (right thigh and top of head) were shaved and cleaned using a betadine scrub and isopropyl alcohol. Its maxillary central incisors were hooked into a gas mask through which it continued to receive small doses of anesthesia. It was secured to a surgery table and its body temperature was regulated with a hot pad. Incisions were made along the right thigh to expose the sciatic nerve, tibialis anterior (TA), and soleus (SOL) muscles. The nerve was severed, proximal to the tibial and fibular nerves, and the $\mu$ PNI was implanted by suturing both the distal and proximal ends of the nerves to the guides of the device (Figure 2). EMG signals were obtained by implanting pairs of microwires (Stablohm 800A, California Fine Wires, CA) $(75 \mu \mathrm{m}$ diameter $)$ into the TA and SOL. All electrodes were guided subcutaneously to an incision made at the top of the head and henceforth attached to a connector (Nano Strip Connector, A79022-001, Omnetics, MN), which was secured to the skull using dental cement and stainless steel screws. All procedures conformed to the Guide for the Care and Use of Laboratory Animals of the Institute of Laboratory Animal Resources, Commission on Life Sciences, National Research Council (National Academy Press, Washington, DC, 1996) and were reviewed and approved by the Institutional Animal Care and Use Committee UTRGV.

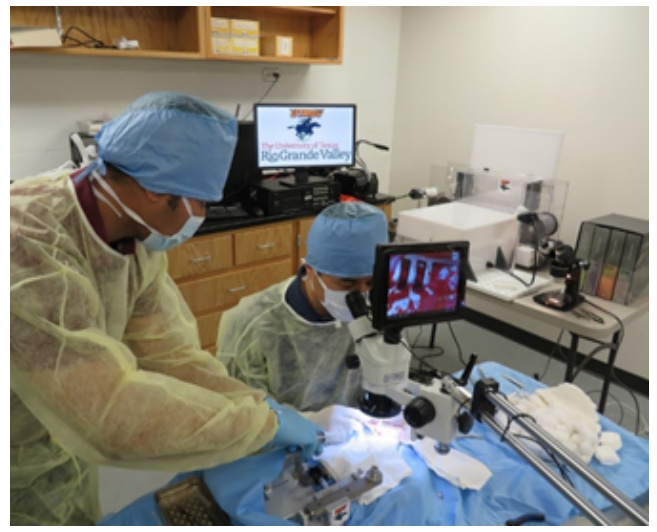

Figure 1: Surgery setup and implementation. All surgical procedures were done under stringent ethical standards at the UTRGV animal facility. 


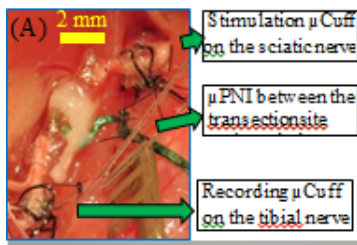

(C)

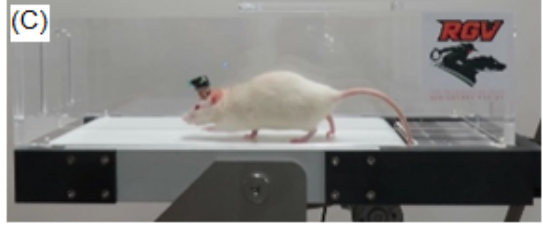

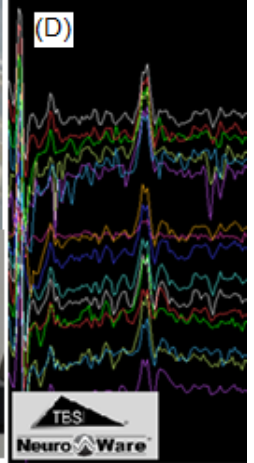

Figure 2: (A) Animal surgery of the sciatic nerve model. Two $\mu$ Cuff electrodes and $\mu \mathrm{PNI}$ are located. (B) Both TBSI stimulation and recording system headstages on a rat. (C) Video monitoring of walking locomotion. 32-channel TBSI wireless recording device is attached on the headplug (D) Neural signals from individual microwires showing different signals from each channel recorded by TBSI 32-channel wireless system. The first picks are the stimulation signals and the second picks are evoked action potentials delayed by the transition speed of the peripheral nerves.

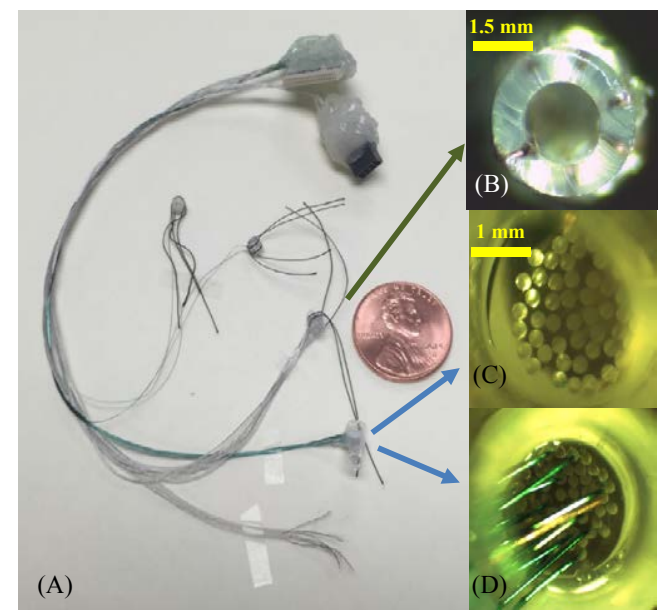

Figure 3: Implantable micro devices (A) whole configuration. (B) 8-electrode $\mu$ Cuff for stimulation. (C) $160 \mu \mathrm{m}$ diameter microchannel scaffold before inserting microwires. 16 out of 50 microchannels were occupied by microwires. (D) 16-electrode $\mu \mathrm{PNI}$ after microwires were inserted inside microchannels.

\section{Results}

A manually fabricated implantable micro device ready for the surgery is shown in Figure 3. Both an omnetics connector (Nano Strip Connector, A79022-001, Omnetics, MN) for TBSI neuroware and a sullins connector (S9009E-04-ND, 8 Position 050, Dual Row, Digi-key) for TBSI stimware were placed at one end of the microwire bundle. The other end of the microwire bundle was connected with a $\mu \mathrm{PNI}$, three $\mu \mathrm{Cuffs}$, and two electromyography electrodes. Peripheral nerve axons were targeted in the $\mu \mathrm{PNI}$ using microchannels that isolate different groups of axons. Since the developed fabrication technique is simple and adjustable, the scaffold parameters (length and microchannel diameter) can be modified to fit different applications. Figure 3C indicates that each microchannel $(160 \mu \mathrm{m}$ diameter $)$ is individually separated and completely sealed. This feature aims to improve the design by reducing the crosstalk between adjacent microchannels and increasing the signal-to-noise ratio. This is a significant advantage of the $\mu \mathrm{PNI}$ because other electrodes that are near each other can create crosstalk due to parasitic capacitances [27].

Figure $2 \mathrm{~A}$ shows the successful surgery result. The $\mu \mathrm{PNI}$ was implanted between the transected sciatic nerve stumps at the location marked as 'transection' of the schematic design in Figure 4. All embedded electrodes were routed subcutaneously and connected to a head-mounted plug (Figure 2B). The nerve stumps were sutured on each side of the $\mu$ PNI. The stimulation $\mu$ Cuff was placed on the proximal sciatic nerve from the $\mu$ PNI. Once the microchannel scaffolds were occupied by the regenerated nerve, the stimulation signal from the $\mu \mathrm{Cuff}$ was recorded from the recording $\mu$ Cuff on the tibial and the common peronial nerves. This confirmed the successful nerve regeneration through the $\mu$ PNI. The $\mu$ Cuff electrodes were used as supplementary recording devices and contributed as a part of neural networks in the sciatic nerve branches. The electrophysiological locomotion data of the sNI was captured by Triangle BioSystems International (TBSI, Durham, NC) wireless system. Figure 2B shows both TBSI w-32 wireless recording system and TBSI S2W stimulator. While an animal was walking on a treadmill for behavioral pattern analysis, TBSI w-32 system recorded the electrophysiological signals from all implanted micro devices (Figure 2C). We used a rodent treadmill system that has the slop angle control capability (760306, Harvard Apparatus, South Natick, Massachusetts), which was installed in a procedure room at the animal facility at UT-RGV. TBSI S2W stimulator was used to generate the evoked signal to analyze the neural pathways and electrophysiological properties of the sciatic nerve branches, SOL, and TA muscles. Neural recording and stimulation signals were forced to flow longitudinally within the microchannel scaffolds which make each microchannel independent from all other microchannels, making it possible to retrieve specific signals. The implantable devices of the $\mu \mathrm{PNI}$, the $\mu \mathrm{Cuff}$, and the EMG electrodes were implanted in the animal and neural signal recordings were obtained, while the animal was running on a treadmill. The TBSI wireless recording system gave the maximum flexibility for the locomotion studies. Due to the robust nerve regeneration of the sciatic nerve model, all channels were occupied with regenerated nerves. Figure 2D shows the electrophysiological signals captured by the 16-electrode microwire $\mu$ PNI using TBSI wireless recording system three weeks after implantation. The neural signals through the regenerated nerves in the $\mu$ PNI were recorded and analyzed to retrieve data corresponding to animal behavior patterns. Electrophysiological signals were recorded from all 16 electrodes. Although some signals showed were identical, these also suggested possible axonal branching from the regenerated nerves. With further analysis in the future work, we could determine if the multiple axons were originated from the same parent neuron to make a same neural signal pattern. It could be confirmed by histology analysis at the end of the procedure after harvesting regenerated nerve tissues. The unique neural signal patterns of the $\mu$ PNI, depending on the animal behavior patterns, will not only confirm the brain-controlled neural singnals at the $\mu \mathrm{PNI}$, but also pioneer the delicate neuronal networks in the brain linked to the sensory and motor feedback of peripheral nerves. Action potentials with similar waveforms were identified in the locomotion microelectrode recordings and extracted using a timeamplitude window discriminator routine. The average amplitude of the action potentials extracted from microchannels was about 100 $\mu \mathrm{V}$ with amplitudes ranging from $40 \sim 200 \mu \mathrm{V}$. Selected and repeated action potentials comparing the $\mu$ PNI, TA muscle, and cuff data were clearly demonstrating the step cycles. A neural signal combination of all microwires of the $\mu \mathrm{PNI}$, or part of them, will express a behavioral pattern at a specific temporal moment. A repeatable behavioral pattern 


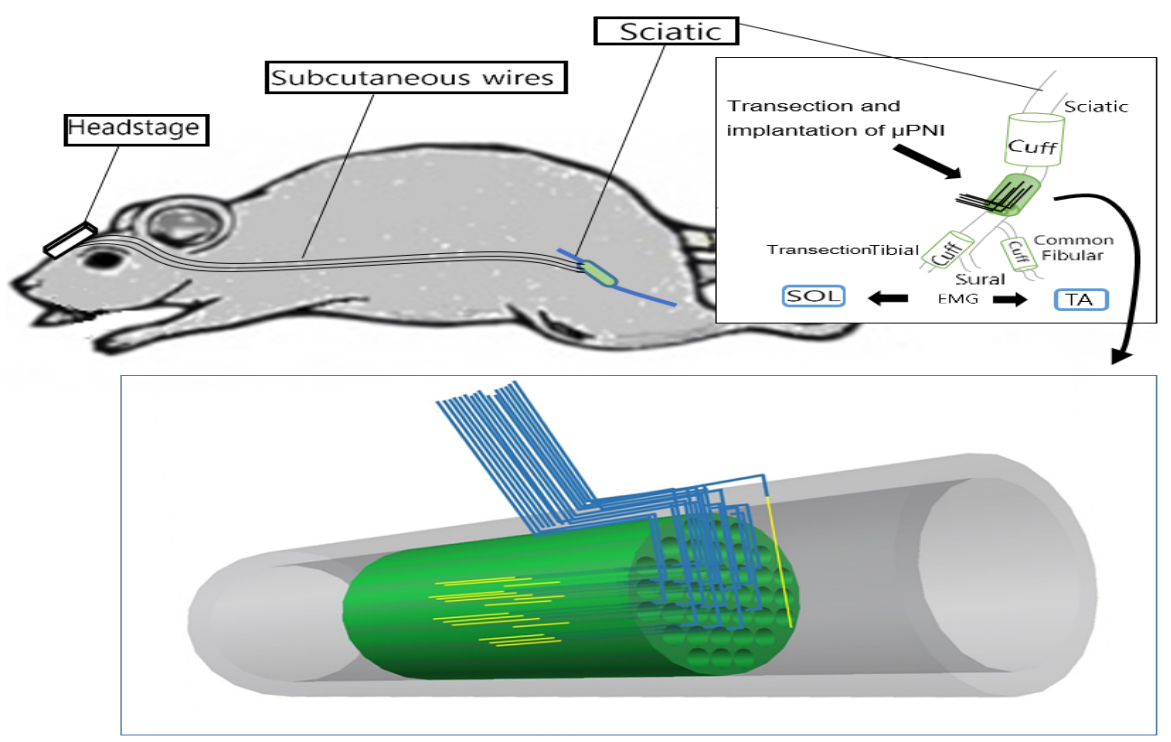

Figure 4: Schematic view of the animal model for $\mu \mathrm{PNI}, \mu$ Cuff, and EMG electrodes on the sciatic nerve braches.

may express a series of temporal neural signal patterns. Thin scar tissue formation covering outside PDMS scaffolds was observed from the harvested $\mu$ PNI. However, no obstructing inflammation responses was observed inside microchannels with a two-month regeneration period. PDMS is an FDA-approved biomaterial for several clinical applications. As a biocompatible material, PDMS has been used in a wide range of applications, such as a structure itself as part of the device and an insulator. PDMS cuff electrodes have been used on the extradural sacral root to sense the bladder response to stimulation in patients [7].

\section{Discussion}

For the chronic animal studies, the microdevices need to be implanted securely inside the animal body without biological rejection and mechanical failure. Moreover, minimally invasive surgery is always required. A biological reaction to foreign materials could be significant in any chronic animal study requiring implantation, especially those that require the implanted device to be kept for more than three months. We recorded electrophysiological signals with the $\mu$ PNI for two months period, since the axonal reinnervation was achieved after one month and stable muscle signals could be captured afterward. Many implantable devices have been used in the everyday clinical practice and decades of implantation is not a significant issue anymore, which makes us confident about the chronic studies. We used PDMS as a base material for all components of the $\mu$ PNI. PDMS has been widely used as a major material of the implantable devices for both research and clinical purposes [28-33], due to its easy fabrication technique and biocompatibility. To achieve translational capabilities, PDMS could be replaced by biodegradable materials, such as PCL, PLGA, and PGA [34-39]. After the nerve regeneration, the biodegradable microchannel will be dissolved to give the structures as close to a natural nerve as possible. Each biodegradable material needs to be tested for its own biocompatibility and degradation rate in the peripheral nerve model.

Though we have developed the $\mu \mathrm{PNI}$ targeting the sciatic nerve model with gait analysis of the somatic nervous system, it can be easily adapted for other nerve models including the modulation of the autonomic nervous system. The developed fabrication technique of the $\mu \mathrm{PNI}$ is not dependent on the nerve size as any size of the $\mu$ PNI components can be developed, ranging from few hundred micrometers to several millimeters in diameter which covers almost all major peripheral nerves and their branches. This allows for flexibility in choosing from a variety of design configurations that target specific nerve fibers.

The $\mu \mathrm{PNI}$ has a significant potential as neuroscience research test beds, if it is combined with biochemical neurotropic factors. The microchannels of the $\mu \mathrm{PNI}$ can be coated with different neurotropic factors to separate the growth of sensory or motor specific axons into the microchannels. They will encapsulate multiple neurotropic factors, such as nerve growth factor (NGF) [40], neurotrophin-3 (NT3) $[41-43]$, brain-derived neurotrophic factor (BDNF) $[44,45]$, and neurotrophin 4/5 (NT-4/5) [46], ciliary neurotrophic factor (CNTF), and glial cell line-derived neurotrophic factor (GDNF). Inducing the specific axonal growth from a microchannel structure to biochemically infused microchannels could provide data for a more in-depth analysis of axon growth behavior.

\section{References}

1. Dhillon GS, Lawrence SM, Hutchinson DT, Horch KW (2004) Residual function in peripheral nerve stumps of amputees: implications for neural control of artificial limbs. J Hand Surg Am 29: 605-615.

2. Kuiken TA, Li G, Lock BA, Lipschutz RD, Miller LA, Stubblefield KA, et al. (2009) Targeted Muscle Reinnervation for Real-Time Myoelectric Control of Multifunction Artificial Arms. JAMA 301: 619-628.

3. Rossini PM, Micera S, Benvenuto A, Carpaneto J, Cavallo G, et al. (2010) Double nerve intraneural interface implant on a human amputee for robotic hand control. Clin Neurophysiol 121: 777-783.

4. Agnew SP, Schultz AE, Dumanian GA, Kuiken TA (2012) Targeted reinnervation in the transfemoral amputee: a preliminary study of surgical technique. Plast Reconstr Surg 129: 187-194.

5. Badia J, Boretius T, Andreu D, Azevedo-Coste C, Stieglitz T, et al. (2011) Comparative analysis of transverse intrafascicular multichannel, longitudinal intrafascicular and multipolar cuff electrodes for the selective stimulation of nerve fascicles. J Neural Eng 8: 036023.

6. Tyler DJ, Durand DM (2002) Functionally selective peripheral nerve stimulation 
Citation: Ajam A, Hossain R, Tasnim N, Castanuela L, Ramos R, et al. (2016) Handcrafted Microwire Regenerative Peripheral Nerve Interfaces with Wireless Neural Recording and Stimulation Capabilities. Sensor Netw Data Commun 5: 133. doi:10.4172/2090-4886.1000133

with a flat interface nerve electrode. IEEE Trans Neural Syst Rehabil Eng 10: 294-303.

7. Grill WM, Mortimer JT (2000) Neural and connective tissue response to longterm implantation of multiple contact nerve cuff electrodes. J Biomed Mater Res 50: 215-226.

8. Serra J, Bostock $H$, Navarro X (2010) Microneurography in rats: a minimally invasive method to record single $\mathrm{C}$-fiber action potentials from peripheral nerves in vivo. Neurosci Lett 470: 168-174

9. Lawrence SM, Dhillon GS, Jensen W, Yoshida K, Horch KW (2004) Acute peripheral nerve recording characteristics of polymer-based longitudinal intrafascicular electrodes. IEEE Trans Neural Syst Rehabil Eng 12: 345-348.

10. Rossini PM, Rigosa J, Micera S, Assenza G, Rossini L, et al. (2011) Stump nerve signals during transcranial magnetic motor cortex stimulation recorded in an amputee via longitudinal intrafascicular electrodes. Experimental Brain Research 210: 1-11.

11. Branner A, Stein RB, Fernandez E, Aoyagi Y, Normann RA (2004) Long-term stimulation and recording with a penetrating microelectrode array in cat sciatic nerve. IEEE Trans Biomed Eng 51: 146-157.

12. Thurgood BK, Warren DJ, Ledbetter NM, Clark GA, Harrison RR (2009) A wireless integrated circuit for 100-channel charge-balanced neural stimulation. IEEE Trans Biomed Circuits Syst 3: 405-414.

13. Normann RA (2007) Technology insight: future neuroprosthetic therapies for disorders of the nervous system. Nat Clin Pract Neurol 3: 444-452.

14. Navarro X, Calvet S, Rodríguez FJ, Stieglitz T, Blau C, et al. (1998) Stimulation and recording from regenerated peripheral nerves through polyimide sieve electrodes. J Peripher Nerv Syst 3: 91-101.

15. Ceballos D, Valero-Cabré A, Valderrama E, Schüttler $M$, Stieglitz $T$, et al (2002) Morphologic and functional evaluation of peripheral nerve fibers regenerated through polyimide sieve electrodes over long-term implantation. J Biomed Mater Res 60: 517-528.

16. Lago N, Ceballos D, Rodríguez FJ, Stieglitz T, Navarro X (2005) Long term assessment of axonal regeneration through polyimide regenerative electrodes to interface the peripheral nerve. Biomaterials 26: 2021-2031.

17. Castro J, Negredo P, Avendaño C (2008) Fiber composition of the rat sciatic nerve and its modification during regeneration through a sieve electrode. Brain Res 1190: 65-77.

18. Cho SH, Lu HM, Cauller L, Romero Ortega MI, Lee JB, et al. (2008) Biocompatible SU-8-Based Microprobes for Recording Neural Spike Signals From Regenerated Peripheral Nerve Fibers. IEEE Sensors Journal 8: 18301836.

19. Garde K, Keefer E, Botterman B, Galvan P, Romero MI (2009) Early interfaced neural activity from chronic amputated nerves. Front Neuroeng 2: 5.

20. FitzGerald JJ, Lago N, Benmerah S, Serra J, Watling CP, et al. (2012) A regenerative microchannel neural interface for recording from and stimulating peripheral axons in vivo. J Neural Eng 9: 016010

21. Gore RK, Choi Y, Bellamkonda R, English A (2015) Functional recordings from awake, behaving rodents through a microchannel based regenerative neural interface. J Neural Eng 12: 016017

22. Choi Y, Gore RK, English AW, Bellamkonda RV (2012) Multilumen PDMS scaffolds for peripheral nerve repair and interface. In: the 40th Neural Interfaces Conference, Salt Lake City, UT, 2012.

23. Choi Y, Park S, Chung Y, Gore RK, English AW, et al. (2014) PDMS microc hannel scaffolds for neural interfaces with the peripheral nervous system. In The 27th IEEE International Conference on Micro Electro Mechanical Systems (MEMS 2014), San Francisco, CA.

24. Choi Y, Shafqat F, Heo H, Bellamkonda RV (2012) Development of micro channel nerve grafts and their application in peripheral nerve regeneration. In: 2012 International Annual Symposium on Regenerative Rehabilitation, Pittsburgh, PA.

25. Gore RK, Choi Y, English AW, Bellamkonda RV (2012) Peripheral neura recordings in awake and behaving rats after sciatic regeneration through a microchannel based neural interface. In: Neuroscience 2012, Society for Neuroscience 42nd annual meeting, New Orleans, LA.
26. Hossain R, Kim B, Pankratz R, Ajam A, Park S, et al. (2015) Handcrafted multilayer PDMS microchannel scaffolds for peripheral nerve regeneration. Biomed Microdevices 17: 109.

27. Takei K, Kawano T, Kawashima T, Sawada K, Kaneko H, et al. (2010) Microtube-based electrode arrays for low invasive extracellular recording with a high signal-to-noise ratio. Biomed Microdevices 12: 41-48.

28. Kurstjens GaM, Borau A, Rodriguez A, Rijkhoff NJM, Sinkjaer T (2005) Intraoperative recording of electroneurographic signals from cuff electrodes on extradural sacral roots in spinal cord injured patients. The Journal of Urology 174: 1482-1487

29. Stöver T, Lenarz T (2009) Biomaterials in cochlear implants. GMS Curr Top Otorhinolaryngol Head Neck Surg 8: Doc10.

30. Malcolm RK, Edwards KL, Kiser P, Romano J, Smith TJ (2010) Advances in microbicide vaginal rings. Antiviral Res 88 Suppl 1: S30-39.

31. Grill WM, Craggs MD, Foreman RD, Ludlow CL, Buller JL (2001) Emerging clinical applications of electrical stimulation: opportunities for restoration of function. J Rehabil Res Dev 38: 641-653.

32. Martens FM, Heesakkers JP (2011) Clinical results of a brindley procedure: sacral anterior root stimulation in combination with a rhizotomy of the dorsa roots. Adv Urol 2011: 709708.

33. Andriot M, Degroot JV, Meek J, Meeks R, Gerlach E, et al (2007) Silicones in Industrial Applications. Inorganic Polymers, RD Jaeger and M. Gleria, Eds ed: Nova Science Publishers pp. 61-161.

34. Sivak WN, Bliley JM, Marra KG (2014) Polymeric Biomaterials for Nerve Regeneration: Fabrication and Implantation of a Biodegradable Nerve Guide. In: Axon Growth and Regeneration A. J. Murray, Ed ed: Springer pp. 139-148.

35. Liu JJ, Wang CY, Wang JG, Ruan HJ, Fan CY (2011) Peripheral nerve regeneration using composite poly (lactic acid-caprolactone)/nerve growth factor conduits prepared by coaxial electrospinning. Journal of Biomedical Materials Research Part A 96A: 13-20.

36. Nectow AR, Marra KG, Kaplan DL (2012) Biomaterials for the development of peripheral nerve guidance conduits. Tissue Eng Part B Rev 18: 40-50.

37. Reid AJ1, de Luca AC, Faroni A, Downes S, Sun M, et al. (2013) Long term peripheral nerve regeneration using a novel PCL nerve conduit. Neurosci Lett 544: $125-130$

38. Kehoe S, Zhang XF, Boyd D (2012) FDA approved guidance conduits and wraps for peripheral nerve injury: a review of materials and efficacy. Injury 43 553-572.

39. Mobasseri A, Faroni A, Minogue BM, Downes S, Terenghi G, et al. (2015) Polymer scaffolds with preferential parallel grooves enhance nerve regeneration. Tissue Eng Part A 21: 1152-1162.

40. Lotfi P, Garde K, Chouhan AK, Bengali E, Romero-Ortega MI (2011) Modalityspecific axonal regeneration: toward selective regenerative neural interfaces. Frontiers In Neuroengineering 4: 11-11.

41. Sterne GD, Brown RA, Green CJ, Terenghi G (1997) Neurotrophin-3 delivered locally via fibronectin mats enhances peripheral nerve regeneration. Eur $J$ Neurosci 9: 1388-1396.

42. Yamauchi J, Chan JR, Miyamoto Y, Tsujimoto G, Shooter EM (2005) The neurotrophin-3 receptor TrkC directly phosphorylates and activates the nucleotide exchange factor Dbs to enhance Schwann cell migration. Proc Nat Acad Sci U S A 102: 5198-5203.

43. Sahenk Z, Oblinger J, Edwards C (2008) Neurotrophin-3 deficient Schwann cells impair nerve regeneration. Exp Neurol 212: 552-556.

44. Utley DS, Lewin SL, Cheng ET, Verity AN, Sierra D, et al. (1996) Brain-derived neurotrophic factor and collagen tubulization enhance functional recovery afte peripheral nerve transection and repair. Arch Otolaryngol Head Neck Surg 122 407-413.

45. Sendtner M, Holtmann B, Kolbeck R, Thoenen H, Barde YA (1992) Brainderived neurotrophic factor prevents the death of motoneurons in newborn rats after nerve section. Nature 360: 757-759.

46. Liu X, Jaenisch R (2000) Severe peripheral sensory neuron loss and modest motor neuron reduction in mice with combined deficiency of brain-derived neurotrophic factor, neurotrophin 3 and neurotrophin 4/5. Dev Dyn 218: 94-101. 